\title{
The Efficacy and Safety of Dexmedetomidine for Sedation During Surgery Under Epidural or Spinal Anesthesia: A Randomized, Double-Blind, Placebo-Controlled Study
}

\author{
Yoshimi Inagaki,* Michiaki Yamakage, $\dagger$ Atsuhiro Sakamoto, \\ Takehiko Hiraokall and Kiyoshi Moritaq \\ *Department of Anesthesiology and Critical Care Medicine, School of Medicine, Faculty of Medicine, Tottori University, Yonago 683- \\ 8504, Japan, †Department of Anesthesiology, Sapporo Medical University School of Medicine, Sapporo 060-8556, Japan, †Department \\ of Anesthesiology, Nippon Medical School, Tokyo 113-8602, Japan, §Clinical Statistics Group 2, Biometrics \& Data Management, \\ Pfizer R\&D Japan, Tokyo 151-8589, Japan, \|Clinical Development Department, Maruishi Pharmaceutical Co., Ltd., Osaka 538-0042, \\ Japan, and $\Phi$ Okayama University Graduate School of Medicine, Dentistry and Pharmaceutical Science, Okayama700-8558, Japan
}

\section{ABSTRACT}

Background Only a few studies have been reported on the use of dexmedetomidine for sedating surgical patients requiring epidural or spinal anesthesia. We conducted a randomized, double-blind, placebo-controlled, parallel-group study at 12 hospitals in Japan.

Methods Adult patients were randomly allocated to receive an intravenous administration of placebo or dexmedetomidine at $0.067,0.25,0.5$ or $1.0 \mu \mathrm{g} / \mathrm{kg}$ over 10 min after epidural or spinal anesthesia. All dexmedetomidine groups received dexmedetomidine $0.2-0.7 \mu \mathrm{g} / \mathrm{kg} / \mathrm{h}$ to maintain an Observer's Assessment of Alertness/Sedation Scale (OAA/S) score of $\leq 4$; however, propofol was administered to rescue patients who exceeded this score. Surgery was then started 15 min after study drug infusion in patients with OAA/S score of $\leq 4$. The primary endpoint was the percentage of patients not requiring rescue propofol to achieve and maintain an OAA/S score of $\leq 4$.

Results Of the 120 enrolled and randomized patients, 119 were treated the study: 22 received placebo and 97 received dexmedetomidine (23-25 patients per dose). Significantly more patients did not require propofol in the dexmedetomidine 0.5 and $1.0 \mu \mathrm{g} / \mathrm{kg}$ groups $(68.0 \%$ and $80.0 \%$, respectively) compared to the placebo group $(22.7 \%)(P=0.003$ and $P<0.001$, respectively). Common adverse events (AEs) were protocol-defined respiratory depression, bradycardia and hypotension. There was no significant difference in the incidence of AEs between the dexmedetomidine and the placebo groups.

Conclusion We concluded that loading doses of 0.5 and $1.0 \mu \mathrm{g} / \mathrm{kg}$ dexmedetomidine, followed by an infusion at a rate of $0.2-0.7 \mu \mathrm{g} / \mathrm{kg} / \mathrm{h}$, provide effective and well-tolerated sedation for surgical patients during epidural or spinal anesthesia.

Clinical trials.gov identifier: NCT01438957

Key words anesthesia; dexmedetomidine; epidural; propofol; spinal
Dexmedetomidine, a centrally acting $\alpha_{2}$ adrenergic receptor agonist, has preferable sedative properties for monitored anesthesia care in patients undergoing various surgical and diagnostic procedures. ${ }^{1}$ It uniquely lacks clinically significant respiratory depression effects, ${ }^{2}$ unlike midazolam ${ }^{3}$ and propofol, ${ }^{4}$ both of which are commonly used as sedatives for monitored anesthesia care. ${ }^{5}$ Dexmedetomidine also has several other proven benefits: it can be titrated to achieve the desired level of sedation ${ }^{2,6}$; it has analgesic-sparing effects that decrease intra- and post-operative opioid use ${ }^{7,8}$; it has a sympatholytic effect, thus reducing tachycardia and hypertension ${ }^{7,9}$; and its use makes it easy to awake patients and obtain their cooperation. ${ }^{2,6,10,11}$ Current product labelling also describes bradycardia, hypotension and transient hypertension. Therefore, it is considered that dexmedetomidine has the potential to become a safe and effective sedative with less respiratory depression in surgical patients requiring local anesthesia.

According to recent practice guidelines for moderate sedation and analgesia in clinical procedures, benzodiazepines and dexmedetomidine are used as sedatives in settings not intended for general anesthesia. ${ }^{5}$ Although midazolam is a first-line sedative, it can induce respiratory depression both as monotherapy ${ }^{12}$ and in combination with opioid analgesics. ${ }^{3,13}$ Although epidural or spinal anesthesia reduced conscious level

Corresponding author: Yoshimi Inagaki, MD, $\mathrm{PhD}$

yinagaki@tottori-u.ac.jp

Received 2021 September 16

Accepted 2021 December 9

Online published 2022 January 4

Abbreviations: AE, adverse event; ASA, American Society of Anesthesiologists; bpm, beats per minute; CI, confidence interval; DBP, diastolic blood pressure; DEX, dexmedetomidine; FAS, full analysis set; NYHA, New York Heart Association; N.A., not applicable; N, number; OAA/S, Observer's Assessment of Alertness/Sedation Scale; SBP, systolic blood pressure; SD, standard deviation; $\mathrm{SpO}_{2}$, oxygen saturation; TEAE, treatment-emergent adverse event; TUR-Bt, Transurethral resection of bladder tumour; TUR-P, Transurethral resection of the prostate; VAS, visual analogue scale 
due to broad suppression of transmission of peripheral perception to central nervous system, ${ }^{14-16}$ a recent meta-analysis revealed that intravenous administration of dexmedetomidine in patients receiving spinal anesthesia did not increase the depth of anesthesia, induce hypotension during anesthesia, or produce excessive post-operative sedation compared with placebo. ${ }^{17}$ Dexmedetomidine also prolonged the duration both of sensory and motor nerve blockade as well as the time to first analgesic request. ${ }^{17}$ However, the efficacy and safety data on the use of dexmedetomidine as a sedative in surgical patients requiring local anesthesia are limited and its optimal dosage for sedation during epidural or spinal anesthesia has not been determined.

In this study, we aimed to evaluate the efficacy and safety of dexmedetomidine as a sedative in Japanese patients undergoing elective surgery with epidural or spinal anesthesia and to investigate the optimal administration regimen of dexmedetomidine during epidural or spinal anesthesia.

\section{SUBJECTS AND METHODS Study design}

A prospective, randomized, placebo-controlled, doubleblind, parallel-group study was conducted at 12 sites in Japan between June and November 2011. The study was designed by representatives of eight participating research centres in collaboration with two pharmaceutical companies, Hospira Japan Co., Ltd. Osaka, Japan, currently Pfizer Japan Inc., Tokyo, Japan and Maruishi Pharmaceutical Co., Ltd. Osaka, Japan. Data were collected by anesthesiologists at each participating site.

The protocol was approved by the institutional review board at each site, and all patients provided written informed consent before enrolment. The study was conducted in compliance with the ethical principles of the Declaration of Helsinki, and according to the requirements of the Japanese Pharmaceutical Affairs Law, Japanese Good Clinical Practice and all relevant regulatory standards.

Finally, the study protocol was registered in Japan Pharmaceutical Information Center Clinical Trial Information (JapicCTI-No. 111519) on 15 June 2011 and was also registered in Clinical trials.gov (NCT01438957) before patient enrolment.

\section{Patient selection}

Patients were included if they required sedation during elective surgery that was expected to last $\geq 30 \mathrm{~min}$ under epidural or spinal anesthesia without intubation under monitored sedation. The main inclusion criteria were as follows: (1) age $\geq 20$ years; (2) American Society of
Anesthesiologists physical status score of I-III at the time of pre-operative diagnosis. The exclusion criteria were as follows: (1) patients who had undergone general anesthesia or had received $\alpha_{2}$ adrenergic agonists or antagonists within 7 days prior to the date of consent; (2) patients with ventilatory failure requiring intubation or a laryngeal mask; (3) patients with central nervous system pathology that could lead to raised intracranial pressure, uncontrolled seizures, or psychiatric disorders and could impair our ability to assess the response to sedation; (4) contraindications to the study drugs, opioids, other $\alpha_{2}$ adrenergic agonists, or other drugs that may be used in the study; (5) patients diagnosed with unstable angina or acute myocardial infarction within six weeks prior to the date of consent; (6) heart rate $<50$ beats per minute (bpm), systolic blood pressure $<90 \mathrm{mmHg}$, or oxygen saturation $<92 \%$ prior to study drug administration; (7) patients with third-degree heart block without a pacemaker; (8) patients who had experienced an increase in alanine transaminase or aspartate transaminase $>2 \times$ the upper normal limit within 2 months prior to consent or a history of hepatic failure; (9) pregnants or lactating women; (10) any symptom or condition that might cause undue risk to the patients if they participated, or might preclude obtaining satisfactory study data; and (11) patients who had received other study drugs within 30 days prior to the date of consent.

\section{Treatment}

The patients were randomly allocated in a 1:1:1:1:1 ratio using a block design to a placebo group or $0.067,0.25,0.5$, or $1.0 \mu \mathrm{g} / \mathrm{kg}$ dexmedetomidine groups (the DEX 0.067 , DEX 0.25, DEX 0.5 and DEX 1.0 groups).

Each treatment group received a loading dose of placebo or $0.067,0.25,0.5$, or $1.0 \mu \mathrm{g} / \mathrm{kg}$ dexmedetomidine over $10 \mathrm{~min}$ followed by a maintenance dose of placebo or $0.2-0.7 \mu \mathrm{g} / \mathrm{kg} / \mathrm{h}$ of dexmedetomidine.

Patients received a 10 to $12 \mathrm{~mL} / \mathrm{kg}$ intravenous infusion of saline or crystalloid fluid for nearly $30 \mathrm{~min}$ before the epidural or spinal anesthesia to minimise the risk of anesthesia-induced hypotension. During fluid administration, the spinal or epidural anesthesia was applied to induce regional anesthesia. When adequate anesthesia was achieved in the presence of systolic blood pressure $\geq 90 \mathrm{mmHg}$ and heart rate $\geq$ $50 \mathrm{bpm}$, fluid administration was stopped, and infusion of the study drug was started. Patients received intravenous administration of the study drug (placebo or dexmedetomidine) over $10 \mathrm{~min}$ via two dosing lines to maintain blinding: the first line delivered a study drug at $0.375 \mathrm{~mL} / \mathrm{kg} / \mathrm{h}$ (to deliver a dose of $0.25,0.5$ or $1.0 \mu \mathrm{g} / \mathrm{kg}$ dexmedetomidine or placebo prepared in 
indistinguishable vials) and the second line delivered a study drug at $0.1 \mathrm{~mL} / \mathrm{kg} / \mathrm{h}$ (to deliver a dose of 0.067 $\mu \mathrm{g} / \mathrm{kg}$ dexmedetomidine or placebo). The maintenance infusion was started immediately after completion of the initial infusion at a rate of $0.1 \mathrm{~mL} / \mathrm{kg} / \mathrm{h}(0.4 \mu \mathrm{g} /$ $\mathrm{kg} / \mathrm{h}$ dexmedetomidine or placebo), and was adjusted between 0.05 and $0.175 \mathrm{~mL} / \mathrm{kg} / \mathrm{h}(0.2-0.7 \mu \mathrm{g} / \mathrm{kg} /$ $\mathrm{h}$ dexmedetomidine or placebo) to maintain a target sedation level of 3 to 4 on the Observer's Assessment of Alertness/Sedation Scale (OAA/S). ${ }^{18}$

Surgery was started immediately at 15 min after starting the study drug infusion in patients whose $\mathrm{OAA} / \mathrm{S}$ score had reached $\leq 4$ once. If the $\mathrm{OAA} / \mathrm{S}$ score was 5 at any time during the maintenance infusion, the maintenance infusion rate was increased to a maximum rate of $0.175 \mathrm{~mL} / \mathrm{kg} / \mathrm{h}(0.7 \mu \mathrm{g} / \mathrm{kg} / \mathrm{h}$ dexmedetomidine or placebo). If this did not improve the OAA/S score, $0.2 \mathrm{mg} / \mathrm{kg}$ of propofol was gradually and intravenously administered, and it could be repeated until the target level of sedation was achieved. If the OAA/S score was $<3$ at any time during the maintenance infusion, the infusion rate was decreased to a minimum rate of 0.05 $\mathrm{mL} / \mathrm{kg} / \mathrm{h}(0.2 \mu \mathrm{g} / \mathrm{kg} / \mathrm{h}$ dexmedetomidine or placebo $)$. The infusion was stopped at the investigator's discretion after the surgery.

We observed and recorded the OAA/S scores, vital signs and oxygen saturation during infusion of the study drug and until $1 \mathrm{~h}$ after stopping the study drug infusion.

As supplemental analgesic, bolus fentanyl was administered intravenously when a patient verbally complained or had clinical symptoms (e.g., perspiration, tachycardia or increased systolic blood pressure). An intravenous bolus of $0.5 \mu \mathrm{g} / \mathrm{kg}$ fentanyl was permitted to treat pain at minimum intervals of $15 \mathrm{~min}$. Treatmentemergent adverse events (TEAEs) were recorded during infusion and for $24 \mathrm{~h}$ after the end of the study drug infusion. Serious TEAEs were followed up for 30 days.

\section{Efficacy and safety evaluation}

The primary efficacy endpoint was the percentage of patients who did not require rescue propofol to achieve and maintain an OAA/S score $\leq 4$ during study drug administration. Secondary endpoints included the followings: (1) the administration frequency of propofol required to achieve and maintain an OAA/S score $\leq 4$, and the time to first rescue propofol dose; (2) the percentage of time spent with OAA/S scores of $\leq 4$ and 3-4 during infusion of the study drug; (3) the percentage of patients who did not require rescue fentanyl and the administration frequency of fentanyl; and (4) the time to achieve an Aldrete score $\geq 8$ and all scoring items $\geq 1$ following discontinuation of the study drug. ${ }^{19}$ Within $24 \mathrm{~h}$ after discontinuation of the study drug, the investigator rated several factors including the ease of maintaining sedation, hemodynamic stability, respiratory stability and patient cooperation, using visual analogue scale (VAS). Patients also rated their satisfaction with sedation and any associated anxiety. Finally, safety was evaluated, focusing on the incidences of hypotension, hypertension, bradycardia, tachycardia, hypoxia, and respiratory depression (as defined in Table 1), as well as postsurgical nausea and vomiting, and abnormal laboratory values.

\section{Sample size}

Assuming an efficacy rate of 55\% in the DEX groups and $10 \%$ in the placebo group, the sample size was estimated to be 20 patients per group to detect a difference $(45 \%)$ between the DEX groups and the placebo group at a two-sided significance level of $5 \%$ with a power of $80 \%$ based on the $\chi^{2}$-test. Allowing for exclusions and drop-outs, we aimed to enrol 24 patients per group (120 patients in total).

\section{Statistical methods}

A closed testing procedure was used to control familywise error rate for multiple comparison in each efficacy endpoint. Comparison between the DEX 1.0

Table 1. Criteria for hemodynamic and respiratory adverse events

\begin{tabular}{|c|c|c|}
\hline Parameter & Measured value & $\begin{array}{l}\text { Adverse event } \\
\text { name }\end{array}$ \\
\hline \multirow{2}{*}{ SBP } & $\begin{array}{c}<80 \mathrm{mmHg} \text { or } \\
\geq 30 \% \text { decrease from baseline }\end{array}$ & Hypotension \\
\hline & $\begin{array}{c}>180 \mathrm{mmHg} \text { or } \\
\geq 30 \% \text { increase from baseline }\end{array}$ & Hypertension \\
\hline DBP & $\begin{array}{l}<50 \mathrm{mmHg} \\
>100 \mathrm{mmHg}\end{array}$ & $\begin{array}{l}\text { Hypotension } \\
\text { Hypertension }\end{array}$ \\
\hline \multirow{2}{*}{ Heart rate } & $\begin{array}{c}<40 \mathrm{bpm} \text { or } \\
\geq 30 \% \text { decrease from baseline }\end{array}$ & Bradycardia \\
\hline & $\begin{array}{c}>120 \mathrm{bpm} \text { or } \\
\geq 30 \% \text { increase from baseline }\end{array}$ & Tachycardia \\
\hline $\begin{array}{l}\text { Respiration } \\
\text { rate }\end{array}$ & $\begin{array}{c}<8 \mathrm{bpm} \text { or } \\
>25 \% \text { decrease from baseline }\end{array}$ & $\begin{array}{l}\text { Respiratory } \\
\text { depression }\end{array}$ \\
\hline $\mathrm{SpO}_{2}$ & $\begin{array}{c}<90 \% \text { or } \\
\geq 10 \% \text { decrease from baseline }\end{array}$ & Hypoxia \\
\hline
\end{tabular}

Regardless of the criteria, any value requiring medical intervention was considered an adverse event. bpm, beats per minute (heart rate) or breaths per minute (respiratory rate); DBP, diastolic blood pressure; $\mathrm{mmHg}$, millimeter mercury; SBP, systolic blood pressure; $\mathrm{SpO}_{2}$, oxygen saturation. 
and placebo groups was performed first, and comparison between the DEX 0.5 and placebo groups was performed subsequently only if a significant difference was observed in the first comparison. Comparisons of the DEX 0.25 and 0.067 groups with the placebo group were subsequently performed in the same manner. Unless a significant difference was observed between two groups, this procedure was discontinued.

For the primary efficacy analysis of the percentage of patients who did not require rescue propofol, and for the secondary efficacy analysis of the percentage of patients who did not require rescue fentanyl, comparison was performed between each DEX group and the placebo group by the Mantel-Haenszel test. The odds ratios and 95\% confidence intervals (CIs) for each comparison of the percentage of patients who did not require propofol were calculated. A dose-response relationship was assessed by the Mantel extension test as a secondary analysis, with setting the placebo as $0 \mu \mathrm{g} / \mathrm{kg}$. For the secondary efficacy analyses of the propofol and fentanyl administration frequency, the percentages of time with OAA/S scores of $\leq 4$ and 3-4 during the study drug infusion, and the patient evaluation of satisfaction and anxiety, comparison between the DEX groups and the placebo group was performed by the Cochran-Mantel-Haenszel test. In addition, for the secondary efficacy analysis of the time to first rescue dose of propofol, the median time and $95 \%$ CI were determined by the Kaplan-Meier method, and the DEX groups were compared to the placebo group using the stratified log-rank test. For the time to reach an Aldrete score $\geq 8$, the median and $95 \%$ CI were determined by group, and comparison between the DEX groups and the placebo group was performed by the stratified logrank test. For the investigator's VAS scores, descriptive statistics of each VAS score were presented by group, and comparison between each DEX group and the placebo group was performed using the Cochran-MantelHaenszel test. All analyses were adjusted by the type of anesthesia (epidural or spinal anesthesia), as appropriate.

Incidences of total and individual TEAEs were reported by group with comparisons between each DEX group and the placebo group based on the $\chi^{2}$ tests. The efficacy and safety analyses were conducted on the full analysis set, defined as all patients who received any study drug. In addition, subgroup analyses were stratified by age ( $<65$ and $\geq 65$ years) and by anesthesia type (epidural or spinal) for the primary ( $\chi^{2}$ tests) and secondary (Wilcoxon two-sample tests) efficacy endpoints, as well as for the safety evaluations. A $p$-value of $<0.05$ was considered statistically significant in all analyses.

\section{RESULTS}

\section{Baseline characteristics}

A total of 120 patients were randomized, though 1 patient in the placebo group did not receive the study drug. The full analysis set therefore comprised 119 patients, who were allocated as follows: 22 received placebo, 23 received $0.067 \mu \mathrm{g} / \mathrm{kg}$ dexmedetomidine, 24 received $0.25 \mu \mathrm{g} / \mathrm{kg}$ dexmedetomidine, and 25 each received 0.5 and $1.0 \mu \mathrm{g} / \mathrm{kg}$ dexmedetomidine (Fig. 1). The baseline characteristics were similar in these groups (Table 2).

\section{Efficacy}

The numbers and percentages of patients who did not require rescue propofol during the study drug infusion, assessed as the primary endpoint, were significantly higher in the DEX 0.5 and 1.0 groups than in the placebo group $(P=0.003$ and $P<0.001$, respectively, Table 3). In addition, a significant linear dose-response relationship was noted in the four DEX groups $(P<$ $0.001)$.

The frequency of propofol administration was significantly less in the DEX $0.25,0.5$ and 1.0 groups than in the placebo group $(P=0.006, P<0.001$ and $P<$ 0.001 , respectively, Table 4$)$. The median time period to first administration of propofol could not be calculated for the DEX 0.5 and 1.0 groups, because few patients in these DEX groups received propofol. The time period to first propofol was significantly longer in the DEX 0.5 and 1.0 groups compared with the placebo group $(P=$ 0.001 and $P<0.001$, respectively, Table 4).

The higher the dexmedetomidine dose, the more rapid the decrease in the OAA/S score. The time period spent with an OAA/S score $\leq 4$ was significantly longer in the DEX 0.5 and 1.0 groups than the placebo group ( $P=0.002$ and $P<0.001$, respectively), while there was no significant difference between the DEX groups and the placebo group at an OAA/S score of 3-4 (Table 5). It took 15, 25, 25 and 50 min for the DEX 1.0, 0.5, 0.25 and 0.067 groups, respectively, on average to maintain $\leq$ 4 for the mean OAA/S score. Compared to the placebo group, the DEX 1.0, 0.5, 0.25 and 0.067 groups showed the first significant decrease in mean OAA/S scores at $10,15,30$ and $40 \mathrm{~min}$, respectively, after starting the infusion $(P=0.001, P=0.002, P=0.004$ and $P=0.015$, respectively). During recovery from sedation, all DEX groups recovered rapidly, although they showed significantly lower OAA/S scores than the placebo group until 30 min after administration.

Few patients also required rescue fentanyl during the study drug infusion (1 in the placebo group, 2 in the DEX 0.67 group, 0 in the DEX 0.25 group and 1 each in the DEX 0.5 and 1.0 groups), indicating that there 


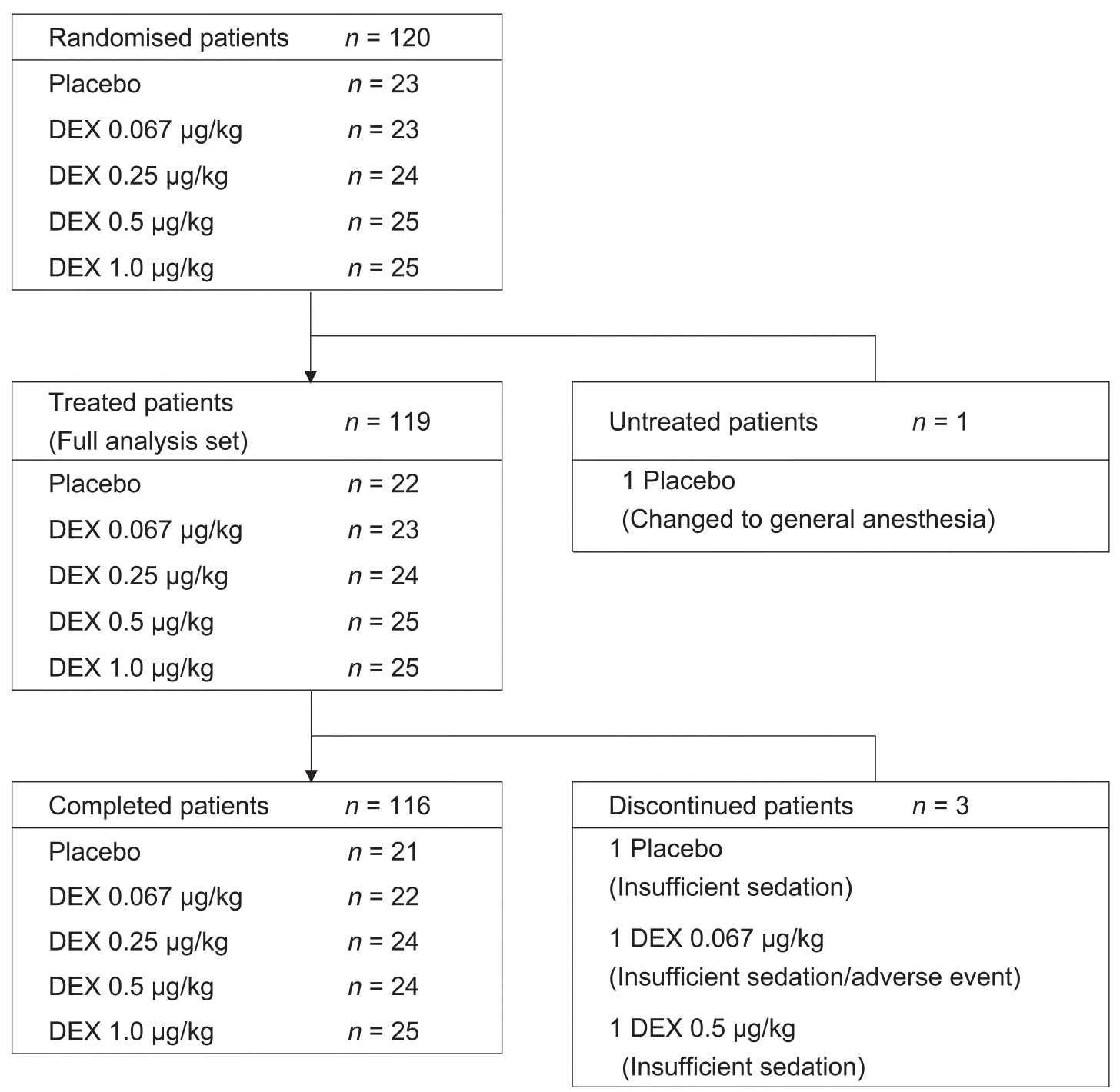

Fig. 1. Study flowchart. Full analysis set was used for efficacy and safety analyses. DEX, dexmedetomidine.

was not a significant difference between the placebo and the DEX 1.0 group. Similarly, the frequency of requiring fentanyl during the study drug infusion was not significantly different between the placebo and DEX 1.0 groups.

The median time to achieve recovery following discontinuation of the study drug, as assessed by an Aldrete score of $\geq 8$, was $15 \mathrm{~min}$ in each treatment group, indicating that there was not a significant difference between the placebo and the DEX 1.0 groups. The 95\% CIs could not be calculated. Ease of maintaining sedation, assessed by the VAS scores, was significantly better in the DEX 0.5 and 1.0 groups than the placebo group $(P=0.014$ and $P=0.005$, respectively), while there was not a significant difference in hemodynamic stability $(P=0.737)$, respiratory stability $(P=0.110)$ or patient cooperation $(P=0.567)$, assessed by the VAS score, between the placebo and the DEX 1.0 groups (Table 6).

The score for patient satisfaction with sedation during surgery under epidural or spinal anesthesia was significantly better in the DEX 1.0 group than the placebo group $(P=0.044$, Table 7$)$. There was not a statistically significant difference between the DEX 1.0 and placebo groups in any other patient-rated measures.

\section{Analyses stratified by age and type of anesthesia}

Stratified analyses were performed by age $(<65$ or $\geq 65$ years) and anesthesia type (epidural or spinal) for the percentage of patients who did not require propofol, the frequency of propofol administration, and the time period spent with an $\mathrm{OAA} / \mathrm{S}$ score $\leq 4$, although the small 
Table 2. Baseline characteristics

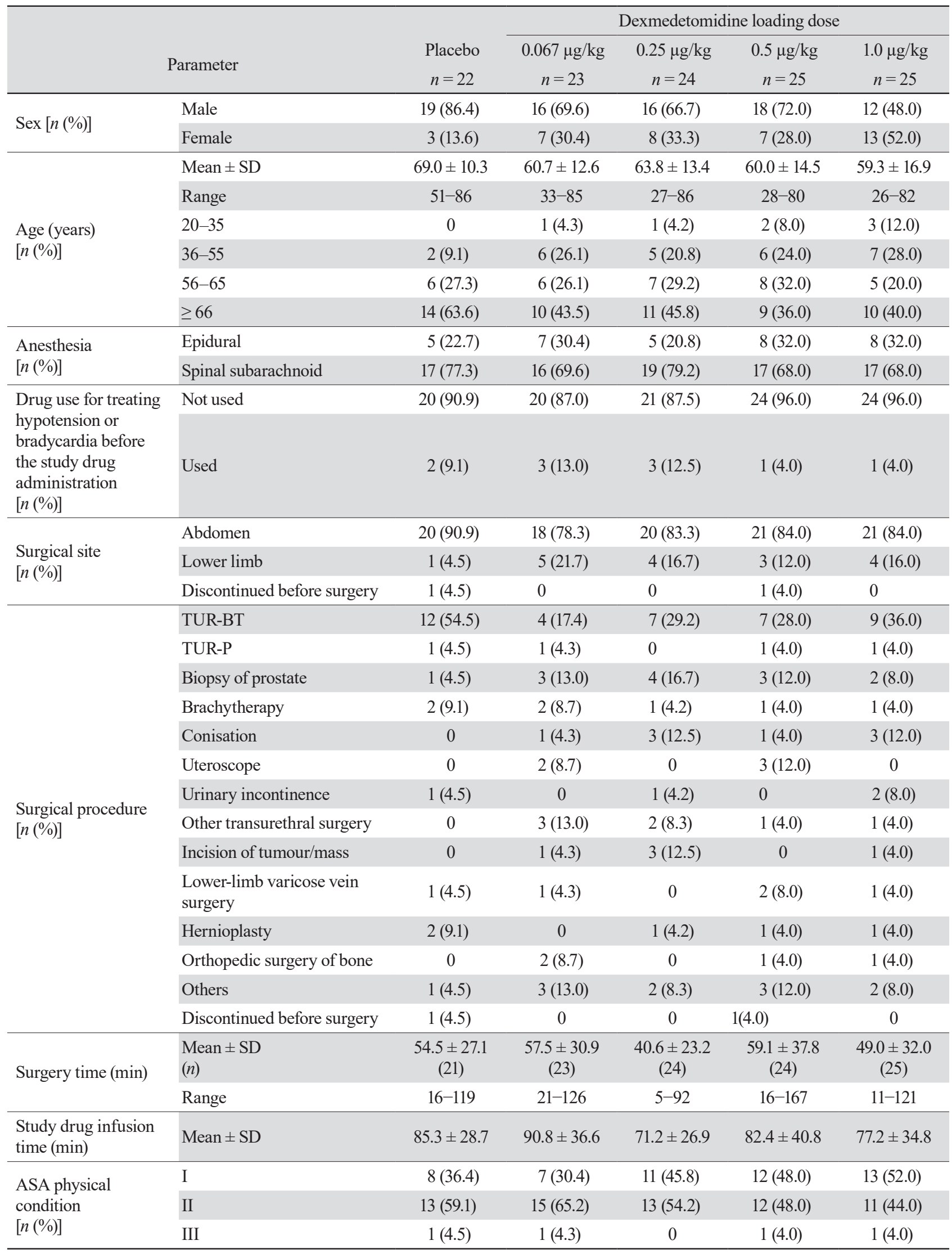


Table 2. Baseline characteristics (Continued)

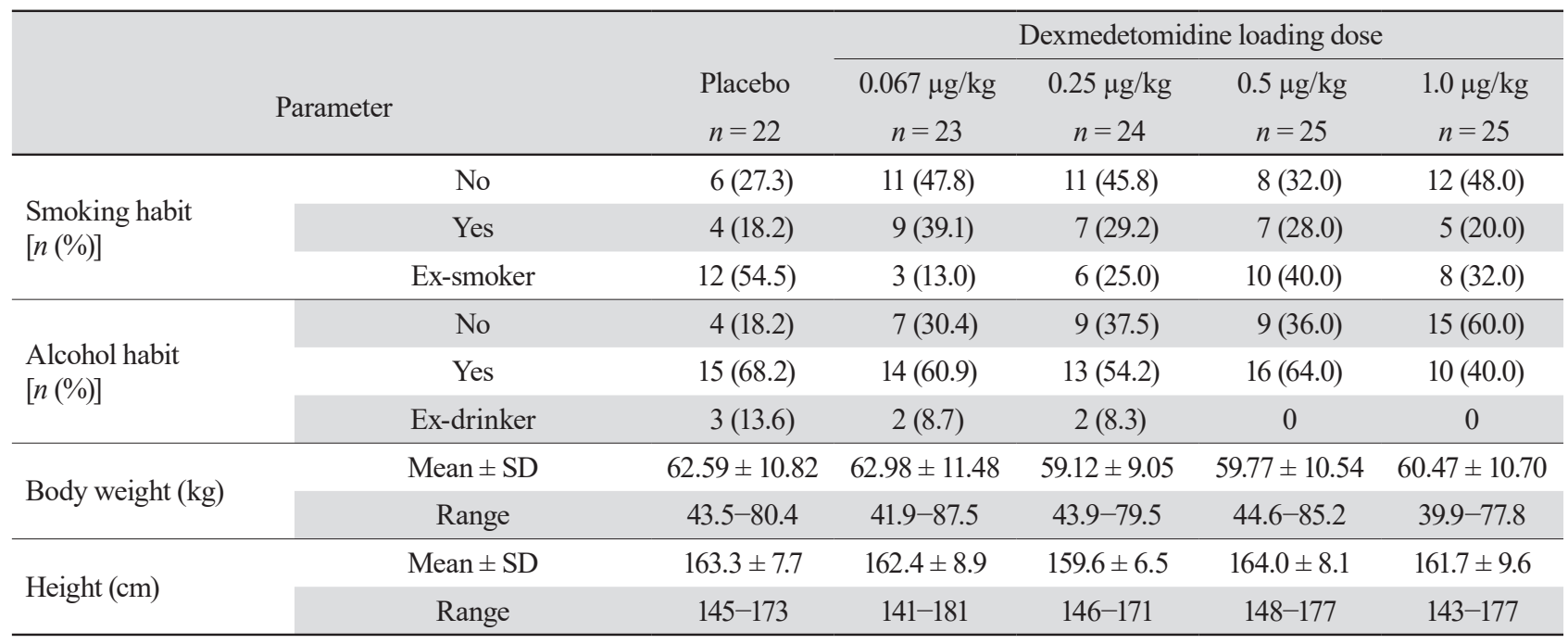

ASA, American Society of Anesthesiologists; Ex-drinker, excessive drinker; Ex-smoker, excessive smoker; SD, standard deviation; TUR-Bt, Transurethral resection of bladder tumour; TUR-P, Transurethral resection of the prostate.

Table 3. Percentages of patients who did not require rescue propofol

\begin{tabular}{|c|c|c|c|c|c|}
\hline & \multirow{3}{*}{$\begin{array}{c}\text { Placebo } \\
n=22\end{array}$} & \multicolumn{4}{|c|}{ Dexmedetomidine loading dose } \\
\hline & & $0.067 \mu \mathrm{g} / \mathrm{kg}$ & $0.25 \mu \mathrm{g} / \mathrm{kg}$ & $0.5 \mu \mathrm{g} / \mathrm{kg}$ & $1.0 \mu \mathrm{g} / \mathrm{kg}$ \\
\hline & & $n=23$ & $n=24$ & $n=25$ & $n=25$ \\
\hline No rescue propofol use $[n(\%)]$ & $5(22.7)$ & $3(13.0)$ & $11(45.8)$ & $17(68.0)$ & $20(80.0)$ \\
\hline $\begin{array}{l}\text { Rescue propofol use } \\
{[n(\%)]}\end{array}$ & $17(77.3)$ & $20(87.0)$ & $13(54.2)$ & $8(32.0)$ & $5(20.0)$ \\
\hline$P$-value* & & - & $P=0.086$ & $P=0.003$ & $P<0.001$ \\
\hline $\begin{array}{l}\text { Odds ratio } † \\
(95 \% \mathrm{CI})\end{array}$ & & $\begin{array}{c}0.510 \\
(0.106-2.453)\end{array}$ & $\begin{array}{c}2.877 \\
(0.800-10.350)\end{array}$ & $\begin{array}{c}7.225 \\
(1.961-26.617)\end{array}$ & $\begin{array}{c}13.600 \\
(3.360-55.045)\end{array}$ \\
\hline $\begin{array}{l}P \text {-value (for dose-response } \\
\text { relationship) }\end{array}$ & & & $P<0.001$ & & \\
\hline
\end{tabular}

*Mantel-Haenszel test adjusted by the type of anesthesia (comparison using a closed testing procedure between the placebo group and each dexmedetomidine group). $\uparrow$ Point estimate and its $95 \%$ confidence limit for the odds ratio of each dexmedetomidine group against the placebo group. \$Mantel-extension test adjusted by the type of anesthesia for detection of a dose-response relationship. CI, confidence interval.

number of patients per stratum limited the analyses. In both age categories, the efficacies of these endpoints were significantly higher in the higher dose of the DEX groups than the placebo group, although we could not compare them fully due to the limited number of cases. For patients under epidural and spinal anesthesia, the limited number of patients who received epidural anesthesia produced equivocal results and no differences were observed in the efficacy by the type of anesthesia (data not shown).

\section{Safety}

Most of the patients experienced at least one TEAE, but they were generally mild (Table 8 ). The most common TEAE was respiratory depression, followed by bradycardia, hypotension, hypertension, hypoxia, glossoptosis and tachycardia. A significant difference was not observed in the overall or individual incidences of TEAEs between the placebo group and any DEX groups. Of the patients who experienced respiratory depression, most of the patients (54/56 across all treatment groups) experienced a protocol-defined decrease in the respiratory rate of $>25 \%$ from baseline, and only seven patients showed a respiratory rate of $<8$ breaths per min. Although six patients (one each in the placebo and DEX 0.25 groups, and two each in the DEX 0.5 and 1.0 groups) required medical intervention, all recovered, and the events were considered clinically insignificant. 
Table 4. Propofol administration during study drug infusion

\begin{tabular}{|c|c|c|c|c|c|}
\hline & \multirow[b]{2}{*}{$\begin{array}{l}\text { Placebo } \\
n=22\end{array}$} & \multicolumn{4}{|c|}{ Dexmedetomidine loading dose } \\
\hline & & $\begin{array}{c}0.067 \mu \mathrm{g} / \mathrm{kg} \\
n=23\end{array}$ & $\begin{array}{c}0.25 \mu \mathrm{g} / \mathrm{kg} \\
n=24\end{array}$ & $\begin{array}{c}0.5 \mu \mathrm{g} / \mathrm{kg} \\
n=25\end{array}$ & $\begin{array}{c}1.0 \mu \mathrm{g} / \mathrm{kg} \\
n=25\end{array}$ \\
\hline \multicolumn{6}{|c|}{ Frequency of rescue propofol administration (times) } \\
\hline Mean \pm SD & $5.1 \pm 4.7$ & $3.7 \pm 3.4$ & $1.9 \pm 2.3$ & $0.8 \pm 1.5$ & $0.5 \pm 1.2$ \\
\hline$P$-value* & & $P=0.329$ & $P=0.006$ & $P<0.001$ & $P<0.001$ \\
\hline \multicolumn{6}{|c|}{ Time to first rescue propofol (min) } \\
\hline Median & 25.5 & 20.0 & 24.0 & - & - \\
\hline $95 \% \mathrm{CI}$ & $20.0-46.0$ & $20.0-22.0$ & $21.0^{-}-$ & $61.0^{-}-$ & --- \\
\hline$P$-valuet & & & $P=0.212$ & $P=0.001$ & $P<0.001$ \\
\hline
\end{tabular}

*Cochran-Mantel-Haenszel test adjusted by the anesthesia type (comparison using a closed testing procedure between the placebo group and each dexmedetomidine group). † Stratified log-rank test adjusted by the type of anesthesia (comparison using a closed testing procedure between the placebo group and each dexmedetomidine group). CI, confidence interval; —, unable to calculate.

Table 5. Percentages of time periods maintained at OAA/S score $\leq 4$ and OAA/S score 3 to 4 during the study drug infusion

\begin{tabular}{|c|c|c|c|c|c|}
\hline & \multirow[b]{2}{*}{$\begin{array}{c}\text { Placebo } \\
n=22\end{array}$} & \multicolumn{4}{|c|}{ Dexmedetomidine loading dose } \\
\hline & & $\begin{array}{c}0.067 \mu \mathrm{g} / \mathrm{kg} \\
n=23\end{array}$ & $\begin{array}{c}0.25 \mu \mathrm{g} / \mathrm{kg} \\
n=24\end{array}$ & $\begin{array}{c}0.5 \mu \mathrm{g} / \mathrm{kg} \\
n=25\end{array}$ & $\begin{array}{c}1.0 \mu \mathrm{g} / \mathrm{kg} \\
n=25\end{array}$ \\
\hline \multicolumn{6}{|l|}{ OAA/S score $\leq 4$} \\
\hline Mean \pm SD (\%) & $50.17 \pm 23.88$ & $58.15 \pm 20.71$ & $61.44 \pm 18.28$ & $70.07 \pm 21.08$ & $76.78 \pm 14.07$ \\
\hline$P$-value* & & & $P=0.116$ & $P=0.002$ & $P<0.001$ \\
\hline \multicolumn{6}{|l|}{ OAA/S score 3 to 4} \\
\hline Mean \pm SD (\%) & $47.88 \pm 25.31$ & $56.00 \pm 19.87$ & $53.66 \pm 17.50$ & $65.30 \pm 20.63$ & $62.25 \pm 23.14$ \\
\hline$P$-value* & & & & & $P=0.088$ \\
\hline
\end{tabular}

*Cochran-Mantel-Haenszel test adjusted by the type of anesthesia (comparison using a closed testing procedure between the placebo group and each dexmedetomidine group).

Hypertension developed in one patient each in the placebo, DEX 0.25 and 0.5 groups, and in six patients in the DEX 1.0 group within 15 min after starting the infusion. Of these, the systolic blood pressure reached $>180 \mathrm{mmHg}$ in one patient who received placebo (no treatment given) and in one patient each in the DEX 0.25 and 1.0 groups (both recovered promptly with nicardipine). The other patients with hypertension did not require treatments. Clinically significant hypertension was not observed during infusion of the initial loading dose. A patient in the DEX 0.067 group developed sepsis during infusion but recovered 5 days later. This was attributed to the surgical procedure and was judged to be unrelated to the study drug. Another patient in the DEX 0.067 group withdrew from the study due to restlessness, which appeared $1 \mathrm{~h}$ after starting the infusion. Because sedation was not achieved with propofol, DEX infusion was stopped and general anesthesia was started instead. This was judged to be "probably not related" to the study drug. Finally, notable differences in the incidences of individual TEAEs were not observed by age ( $<65$ or $\geq 65$ years) or by type of anesthesia (epidural or spinal).

\section{DISCUSSION}

The purpose of this study was to evaluate the efficacy and safety of dexmedetomidine for sedating patients undergoing elective surgery or diagnostic procedures under epidural or spinal anesthesia, and to determine the optimal administration regimen of dexmedetomidine. As judged by the primary efficacy endpoint (percentage of patients who did not require rescue propofol to achieve and maintain an OAA/S score of $\leq 4$ ), a significant sedative effect was observed in the DEX 0.5 and 1.0 groups compared with the placebo group. Higher initial loading doses of dexmedetomidine induced more rapid 
Table 6. Investigator evaluations

\begin{tabular}{|c|c|c|c|c|c|}
\hline & \multicolumn{5}{|c|}{ Dexmedetomidine loading dose } \\
\hline & $\begin{array}{l}\text { Placebo } \\
(n=22)\end{array}$ & $\begin{array}{c}0.067 \mu \mathrm{g} / \mathrm{kg} \\
(n=23)\end{array}$ & $\begin{array}{c}0.25 \mu \mathrm{g} / \mathrm{kg} \\
(n=24)\end{array}$ & $\begin{array}{c}0.5 \mu \mathrm{g} / \mathrm{kg} \\
(n=25)\end{array}$ & $\begin{array}{c}1.0 \mu \mathrm{g} / \mathrm{kg} \\
(n=25)\end{array}$ \\
\hline \multicolumn{6}{|c|}{ Ease of maintaining the sedation level } \\
\hline Mean \pm SD (VAS) & $5.85 \pm 3.54$ & $4.36 \pm 3.41$ & $3.73 \pm 3.19$ & $2.81 \pm 2.64$ & $2.65 \pm 2.98$ \\
\hline$P$-value* & & & $P=0.055$ & $P=0.014$ & $P=0.005$ \\
\hline \multicolumn{6}{|l|}{ Hemodynamic stability } \\
\hline Mean \pm SD (VAS) & $2.05 \pm 2.21$ & $3.30 \pm 3.19$ & $1.68 \pm 1.46$ & $1.72 \pm 2.14$ & $1.47 \pm 1.41$ \\
\hline$P$-value* & & & & & $P=0.737$ \\
\hline \multicolumn{6}{|l|}{ Respiratory stability } \\
\hline Mean \pm SD (VAS) & $2.35 \pm 2.77$ & $2.62 \pm 2.79$ & $1.80 \pm 2.02$ & $2.85 \pm 2.90$ & $3.12 \pm 2.13$ \\
\hline$P$-value* & & & & & $P=0.110$ \\
\hline \multicolumn{6}{|c|}{ Subject's cooperativeness } \\
\hline Mean \pm SD (VAS) & $1.48 \pm 1.67$ & $1.80 \pm 2.35$ & $1.05 \pm 1.46$ & $1.15 \pm 1.19$ & $1.17 \pm 1.46$ \\
\hline$P$-value* & & & & & $P=0.567$ \\
\hline
\end{tabular}

*Cochran-Mantel-Haenszel test adjusted by the type of anesthesia (comparison using a closed testing procedure between the placebo group and each dexmedetomidine group). VAS, visual analogue scale. VAS score ranges from 0 to 10 , with 0 as the most favourable score. Anchors for ease of maintaining sedation level were $0=$ not difficult at all and $10=$ extremely difficult; for hemodynamic stability and respiratory stability were $0=$ very stable and $10=$ extremely unstable; and for patient's cooperation were $0=$ very cooperative and $10=$ extremely uncooperative.

sedation to reach an OAA/S score of $\leq 4$. Although a comparative analysis was not performed among the four dose groups, the sedative effect of dexmedetomidine based on the primary efficacy endpoint also appeared to be dose dependent. The initial loading dose of dexmedetomidine was key to both the induction and maintenance of sedation. Overall, across all treatment groups, a loading dose of $0.5-1.0 \mu \mathrm{g} / \mathrm{kg}(3-6 \mu \mathrm{g} / \mathrm{kg} /$ h) for $10 \mathrm{~min}$ was desirable to induce and maintain appropriate sedation $(\mathrm{OAA} / \mathrm{S} \leq 4)$ for surgery with a mean duration of 40.6-59.1 min (Table 2). Stratified analysis by age indicated that dexmedetomidine was effective at a loading dose of $1.0 \mu \mathrm{g} / \mathrm{kg}$ in both age categories of patients of $<65$ and $\geq 65$ years. However, we could not compare them fully to examine the differences in the effect of dexmedetomidine in both groups due to the limited number of patients.

During recovery from sedation, the DEX groups showed significantly lower OAA/S values compared with the placebo group until 30 min after administration, but all groups recovered similarly thereafter. However, this finding would be due to a lower OAA/S score by approximately 1.0 in the DEX groups than in the placebo group during the study drug infusion. Considering a relatively long elimination half-life of dexmedetomidine (about $3.1 \mathrm{~h}$ ), ${ }^{20}$ the recovery from sedation after discontinuation of dexmedetomidine is considered to be rapid.

Although dexmedetomidine has been shown to have an analgesic-sparing effect, ${ }^{7,8}$ we failed to demonstrate this effect in the present study. This was likely because most of the patients obtained adequate analgesia through epidural or spinal anesthesia. The analgesic effect of dexmedetomidine is probably more relevant under unstable analgesic conditions, such as under topical anesthesia for gastroenteric endoscopy. ${ }^{21,22}$

Although we observed many AEs, there were no significant differences in the overall or individual incidences of TEAEs between the groups. The TEAEs included hypotension, bradycardia and hypertension, which may be induced by the pharmacological action of dexmedetomidine. The high incidence of hemodynamic TEAEs may be due to the conservative protocol-based criteria, the presence of an additive effect between dexmedetomidine and anesthesia (particularly the high incidences of hypotension and bradycardia) or both. ${ }^{23}$ Nonetheless, no events of hypotension or bradycardia were clinically significant in any groups. A comparable incidence of hypotension was also reported in the spinal anesthesia setting between placebo and DEX infusion groups. ${ }^{17}$ Hypertension had been observed after high loading doses of dexmedetomidine in a previous study, ${ }^{6}$ and also in this study it developed relatively more often within 15 min after starting the infusion in the DEX 1.0 
Table 7. Patient satisfaction and anxiety evaluation

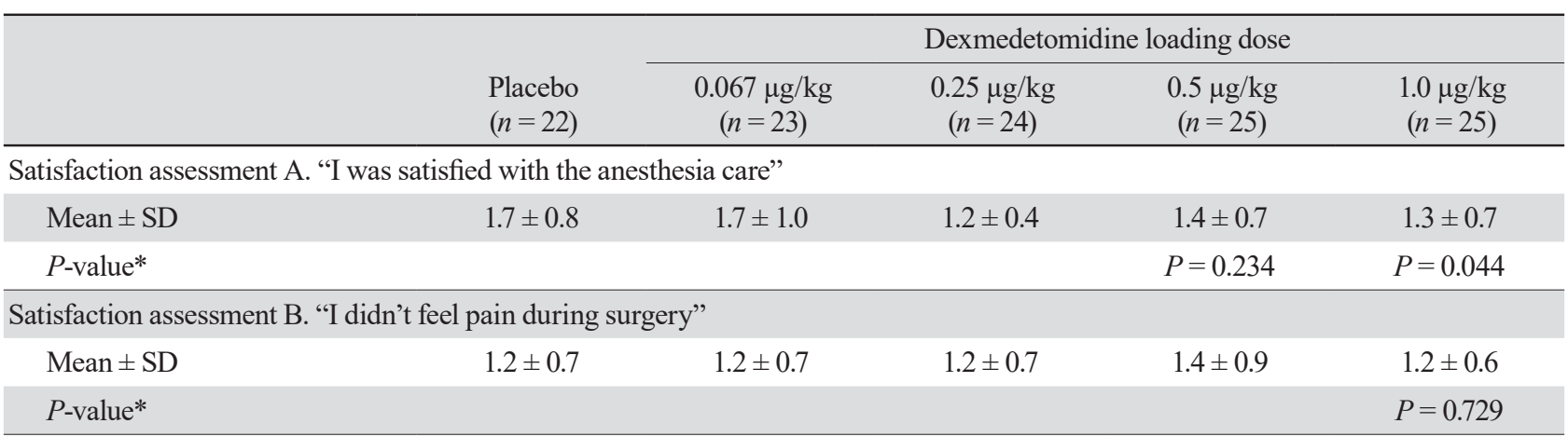

Satisfaction assessment C. "If I were to have similar surgery performed in the future, I would like to have this anesthetic used"

\begin{tabular}{|c|c|c|c|c|c|}
\hline Mean \pm SD & $1.7 \pm 1.1$ & $1.5 \pm 0.8$ & $1.3 \pm 0.6$ & $1.5 \pm 0.8$ & $1.2 \pm 0.4$ \\
\hline$P$-value* & & & & & $P=0.082$ \\
\hline Mean \pm SD & $2.2 \pm 1.3$ & $2.2 \pm 1.3$ & $2.2 \pm 1.2$ & $1.8 \pm 1.0$ & $2.0 \pm 1.2$ \\
\hline$P$-value* & & & & & $P=0.451$ \\
\hline
\end{tabular}

Anxiety assessment E. Assessment of anxiety during surgery.

\begin{tabular}{|c|c|c|c|c|c|}
\hline Mean \pm SD & $1.6 \pm 1.1$ & $1.6 \pm 0.8$ & $1.6 \pm 0.9$ & $1.4 \pm 0.9$ & $1.7 \pm 1.1$ \\
\hline$P$-value* & & & & & $P=0.873$ \\
\hline \multicolumn{6}{|c|}{ Anxiety assessment F. Assessment of anxiety after surgery. } \\
\hline Mean \pm SD & $1.6 \pm 0.9$ & $1.6 \pm 1.0$ & $1.6 \pm 0.6$ & $1.3 \pm 0.7$ & $1.5 \pm 1.0$ \\
\hline$P$-value* & & & & & $P=0.374$ \\
\hline
\end{tabular}

For satisfaction assessments A, B, and C, patients rated satisfaction using a 4-point Likert scale of "agree" (=1), "agree slightly" (=2), "disagree slightly" (=3), or "disagree" (=4). For anxiety assessments D, E, and F, patients rated anxiety using a 5-point numerical rating scale anchored by $1=$ no anxiety and $5=$ extreme anxiety. *Cochran-Mantel-Haenszel test adjusted by the anesthesia type (comparison using a closed testing procedure between the placebo group and each dexmedetomidine group).

Table 8. Treatment-emergent adverse events that occurred with an incidence of $\geq 10 \%$ in any treatment group

\begin{tabular}{|c|c|c|c|c|c|}
\hline & \multirow[b]{2}{*}{$\begin{array}{l}\text { Placebo } \\
(n=22)\end{array}$} & \multicolumn{4}{|c|}{ Dexmedetomidine loading dose } \\
\hline & & $\begin{array}{c}0.067 \mu \mathrm{g} / \mathrm{kg} \\
(n=23)\end{array}$ & $\begin{array}{c}0.25 \mu \mathrm{g} / \mathrm{kg} \\
(n=24)\end{array}$ & $\begin{array}{c}0.5 \mu \mathrm{g} / \mathrm{kg} \\
(n=25)\end{array}$ & $\begin{array}{c}1.0 \mu \mathrm{g} / \mathrm{kg} \\
(n=25)\end{array}$ \\
\hline Number of patients with events (\%) & $\begin{array}{c}19 \\
(86.4)\end{array}$ & $\begin{array}{c}21 \\
(91.3)\end{array}$ & $\begin{array}{c}24 \\
(100.0)\end{array}$ & $\begin{array}{c}22 \\
(88.0)\end{array}$ & $\begin{array}{c}25 \\
(100.0)\end{array}$ \\
\hline Respiratory depression & $\begin{array}{c}12 \\
(54.5)\end{array}$ & $\begin{array}{c}11 \\
(47.8)\end{array}$ & $\begin{array}{c}12 \\
(50.0)\end{array}$ & $\begin{array}{c}10 \\
(40.0)\end{array}$ & $\begin{array}{c}11 \\
(44.0)\end{array}$ \\
\hline Bradycardia & $\begin{array}{c}8 \\
(36.4)\end{array}$ & $\begin{array}{c}11 \\
(47.8)\end{array}$ & $\begin{array}{c}9 \\
(37.5)\end{array}$ & $\begin{array}{c}10 \\
(40.0)\end{array}$ & $\begin{array}{c}11 \\
(44.0)\end{array}$ \\
\hline Hypotension & $\begin{array}{c}5 \\
(22.7)\end{array}$ & $\begin{array}{c}12 \\
(52.2)\end{array}$ & $\begin{array}{c}11 \\
(45.8)\end{array}$ & $\begin{array}{c}8 \\
(32.0)\end{array}$ & $\begin{array}{c}10 \\
(40.0)\end{array}$ \\
\hline Hypertension & $\begin{array}{c}5 \\
(22.7)\end{array}$ & $\begin{array}{c}2 \\
(8.7)\end{array}$ & $\begin{array}{c}2 \\
(8.3)\end{array}$ & $\begin{array}{c}3 \\
(12.0)\end{array}$ & $\begin{array}{c}8 \\
(32.0)\end{array}$ \\
\hline Hypoxia & $\begin{array}{c}4 \\
(18.2)\end{array}$ & $\begin{array}{c}3 \\
(13.0)\end{array}$ & $\begin{array}{c}2 \\
(8.3)\end{array}$ & $\begin{array}{c}3 \\
(12.0)\end{array}$ & $\begin{array}{c}3 \\
(12.0)\end{array}$ \\
\hline Glossoptosis & $\begin{array}{c}3 \\
(13.6)\end{array}$ & 0 & 0 & $\begin{array}{c}2 \\
(8.0)\end{array}$ & $\begin{array}{c}2 \\
(8.0)\end{array}$ \\
\hline Tachycardia & $\begin{array}{c}2 \\
(9.1)\end{array}$ & $\begin{array}{c}3 \\
(13.0)\end{array}$ & 0 & 0 & 0 \\
\hline
\end{tabular}


group, albeit it was not of clinical significance.

The high incidence of respiratory depression in our study could have resulted from the strict criteria as well as the use of propofol in some patients. Most events of respiratory depression were protocol-defined respiratory depression. There was not a significant difference in the incidence of TEAEs by age ( $<65$ and $\geq 65$ years), indicating that dexmedetomidine can safely be used in young and old patients alike.

Candiotti et al. had obtained findings largely consistent with ours. ${ }^{10}$ They showed that dexmedetomidine loading doses of 0.5 and $1.0 \mu \mathrm{g} / \mathrm{kg}$ provided significantly better results than placebo in terms of the proportion of patients who did not require a rescue sedative (midazolam), and the amount of rescue sedative needed to achieve and maintain an OAA/S score of $\leq 4$. Meanwhile, although our study did not show any differences in fentanyl use between the placebo group and each DEX group, Candiotti et al. showed that both DEX groups required significantly less fentanyl than the placebo group. ${ }^{10}$ This difference may have resulted from the epidural and spinal anesthesia providing more effective analgesia than the local blocks. Nevertheless, both studies indicated that dexmedetomidine loading doses of 0.5 and $1.0 \mu \mathrm{g} / \mathrm{kg}$ significantly increased the ease of maintaining the targeted sedation. In our study, clinically significant respiratory depression was observed in all treatment groups (54.5\% in the placebo group, $40.0 \%$ in the DEX 0.5 group and $44.0 \%$ in the DEX 1.0 group), whereas Candiotti et al. showed that the overall incidence of respiratory depression was low and also lower in the DEX groups $(3.7 \%$ in the DEX $0.5 \mu \mathrm{g} / \mathrm{kg}$ group and $2.3 \%$ in the DEX $1.0 \mu \mathrm{g} / \mathrm{kg}$ group) compared with the placebo group (12.7\%). ${ }^{10}$ The likely explanation for this difference is that respiratory depression in our study was defined as a decrease of $>25 \%$ in respiratory rate from baseline, which was a stricter criterion than that in their study. We deemed that our strict criterion for respiratory depression, combined with the differences in anesthesia, were probably responsible for the increased rate of respiratory depression in our study.

As a limitation, because the sample size of this study was small, the finding of subgroup analysis should also be re-evaluated by a large-scale study in the future.

In patients undergoing surgery under epidural or spinal anesthesia, dexmedetomidine infused at loading doses of 0.5 and $1.0 \mu \mathrm{g} / \mathrm{kg}$ over $10 \mathrm{~min}$, followed by a maintenance infusion of $0.2-0.7 \mu \mathrm{g} / \mathrm{kg} / \mathrm{h}$, was significantly superior to placebo to induce sedation and maintain a sedation score of $\leq 4$ on the OAA/S scale. We conclude that dexmedetomidine is an effective and well-tolerated agent for patient sedation during surgery with epidural or spinal anesthesia, regardless of age.

Acknowledgments: This study was funded by Hospira Japan Co., Ltd., Inc. which was acquired by Pfizer Inc. in September 2015. We thank Dr. Ikuto Yoshiya and Dr. Minoru Nomura for assisting with the design of the study and interpretation of the data, and Dr. Toshiro Tango, CIMIC Co., Ltd. for developing the statistical analysis methods. The support provided by WysiWyg Co., Ltd., funded by Pfizer, consisted solely of manuscript formatting and no contribution was made to the editorial content.

\section{CONFLICTS OF INTEREST}

This study was funded by Hospira Japan Co., Ltd., Inc. which was acquired by Pfizer Inc. in September 2015. Pfizer Japan Inc. and Pharmaceutical Co., Ltd. co-developed dexmedetomidine. The data management and statistical analysis services were conducted by CIMIC Co., Ltd. and were funded by Pfizer. YI, MY, $A S, N O, T H$, and KM declare no conflicts of interest. $A O$ is an employee of Pfizer.

\section{THE RESEARCH GROUP}

The study group comprised the three authors, YI, MY, and AS, and the following individuals: Hiroshi Iwasaki, Department of Anesthesiology and Critical Care Medicine, Asahikawa Medical University Hospital; Mikito Kawamata, Department of Anesthesiology and Resuscitology, Shinshu University Hospital; Hironaka Tsunobuchi, Department of Anesthesiology, Nagoya University Hospital; Satoshi Yokono, Department of Anesthesiology, Japanese Red Cross Kyoto Daini Hospital; Takehiko Adachi, Department of Anesthesiology, Kitano Hospital; Kenji Sato, Department of Anesthesiology and Resuscitology, Okayama University Hospital; Yoji Saito, Department of Anesthesiology, Shimane University Hospital; Koichi Yamashita, Department of Anesthesiology and Intensive Care Medicine, Kochi Medical School Hospital; Isao Tsuneyoshi, Department of Anesthesiology, University of Miyazaki Hospital.

\section{DATA-SHARING STATEMENT}

Upon request, and subject to certain criteria, conditions and exceptions (see https://www.pfizer.com/science/clinical-trials/ trial-data-and-results for more information), Pfizer will provide access to individual de-identified participant data from Pfizersponsored global interventional clinical studies conducted for medicines, vaccines and medical devices (1) for indications that have been approved in the USA and/or EU, or (2) in programs that have been terminated (i.e., development for all indications has been discontinued). Pfizer will also consider requests for the protocol, data dictionary, and statistical analysis plan. Data may be requested from Pfizer trials 24 months after study completion. The de-identified participant data will be made available to researchers whose proposals meet the research criteria and other conditions, and for which an exception does not apply, via a secure portal. To gain access, data requestors must enter into a data access agreement with Pfizer. 


\section{REFERENCES}

1 Shukry M, Miller JA. Update on dexmedetomidine: use in nonintubated patients requiring sedation for surgical procedures. Ther Clin Risk Manag. 2010;6:111-21. DOI: 10.2147/ TCRM.S5374, PMID: 20421911

2 Hall JE, Uhrich TD, Barney JA, Arain SR, Ebert TJ. Sedative, amnestic, and analgesic properties of small-dose dexmedetomidine infusions. Anesth Analg. 2000;90:699-705. DOI: 10.1097/00000539-200003000-00035, PMID: 10702460

3 Bailey PL, Pace NL, Ashburn MA, Moll JWB, East KA, Stanley TH. Frequent hypoxemia and apnea after sedation with midazolam and fentanyl. Anesthesiology. 1990;73:82630. DOI: 10.1097/00000542-199011000-00005, PMID: 2122773

4 Blouin RT, Seifert HA, Babenco HD, Conard PF, Gross JB. Propofol depresses the hypoxic ventilatory response during conscious sedation and isohypercapnia. Anesthesiology. 1993;79:1177-82. DOI: 10.1097/00000542-199312000-00007, PMID: 8267192

5 Practice Guidelines for Moderate Procedural Sedation and Analgesia. Practice Guidelines for Moderate Procedural Sedation and Analgesia 2018. Anesthesiology. 2018;128:437-79. DOI: 10.1097/ALN.0000000000002043, PMID: 29334501

6 Ebert TJ, Hall JE, Barney JA, Uhrich TD, Colinco MD. The effects of increasing plasma concentrations of dexmedetomidine in humans. Anesthesiology. 2000;93:382-94. DOI: 10.1097/00000542-200008000-00016, PMID: 10910487

7 Arain SR, Ebert TJ. The efficacy, side effects, and recovery characteristics of dexmedetomidine versus propofol when used for intraoperative sedation. Anesth Analg. 2002;95:4616. DOI: 10.1213/00000539-200208000-00042, PMID: 12145072

8 Elbaradie S, El Mahalawy FH, Solyman AH. Dexmedetomidine vs. propofol for short-term sedation of postoperative mechanically ventilated patients. J Egypt Natl Canc Inst. 2004;16:153-8. PMID: 15959548

9 Abdalla MIM, Mansouri FA, Bener A. Dexmedetomidine during local anesthesia. J Anesth. 2006;20:54-6. DOI: 10.1007/s00540-005-0351-z, PMID: 16421680

10 Candiotti KA, Bergese SD, Bokesch PM, Feldman MA, Wisemandle W, Bekker AY; MAC Study Group. Monitored anesthesia care with dexmedetomidine: a prospective, randomized, double-blind, multicenter trial. Anesth Analg. 2010;110:47-56. DOI: 10.1213/ane.0b013e3181ae0856, PMID: 19713256

11 Kamibayashi T, Maze M, Weiskopf RB, Weiskopf RB, Todd MM. Clinical uses of $\alpha 2$-adrenergic agonists. Anesthesiology. 2000;93:1345-9. DOI: 10.1097/00000542-20001100000030, PMID: 11046225

12 Forster A, Gardaz JP, Suter PM, Gemperle M. Respiratory depression by midazolam and diazepam. Anesthesiology. 1980;53:494-7. DOI: 10.1097/00000542-198012000-00010, PMID: 7457966
13 Cho JS, Shim JK, Na S, Park I, Kwak YL. Improved sedation with dexmedetomidine-remifentanil compared with midazolam-remifentanil during catheter ablation of atrial fibrillation: a randomized, controlled trial. Europace. 2014;16:1000-6. DOI: 10.1093/europace/eut365, PMID: 24381332

14 Hodgson PS, Liu SS. Epidural lidocaine decreases sevoflurane requirement for adequate depth of anesthesia as measured by the Bispectral Index monitor. Anesthesiology. 2001;94:799-803. DOI: 10.1097/00000542-200105000-00018, PMID: 11388531

15 Ishiyama T, Kashimoto S, Oguchi T, Yamaguchi T, Okuyama K, Kumazawa T. Epidural ropivacaine anesthesia decreases the bispectral index during the awake phase and sevoflurane general anesthesia. Anesth Analg. 2005;100:728-32. DOI: 10.1213/01.ANE.0000159868.06847.47, PMID: 15728060

16 Ozkan-Seyhan T, Sungur MO, Senturk E, Karadeniz M, Basel A, Senturk M, et al. BIS guided sedation with propofol during spinal anaesthesia: influence of anaesthetic level on sedation requirement. Br J Anaesth. 2006;96:645-9. DOI: 10.1093/bja/ael055, PMID: 16531442

17 Abdallah FW, Abrishami A, Brull R. The facilitatory effects of intravenous dexmedetomidine on the duration of spinal anesthesia: a systematic review and meta-analysis. Anesth Analg. 2013;117:271-8. DOI: 10.1213/ANE.0b013e318290c566, PMID: 23632057

18 Chernik DA, Gillings D, Laine H, Hendler J, Silver JM, Davidson AB, et al. Validity and reliability of the Observer's Assessment of Alertness/Sedation Scale: study with intravenous midazolam. J Clin Psychopharmacol. 1990;10:244-51. PMID: 2286697

19 Aldrete JA. The post-anesthesia recovery score revisited. J Clin Anesth. 1995;7:89-91. DOI: 10.1016/0952-8180(94)00001K, PMID: 7772368

20 Venn RM, Karol MD, Grounds RM. Pharmacokinetics of dexmedetomidine infusions for sedation of postoperative patients requiring intensive care. Br J Anaesth. 2002;88:66975. DOI: 10.1093/bja/88.5.669, PMID: 12067004

21 Demiraran Y, Korkut E, Tamer A, Yorulmaz I, Kocaman $\mathrm{B}$, Sezen $\mathrm{G}$, et al. The comparison of dexmedetomidine and midazolam used for sedation of patients during upper endoscopy: A prospective, randomized study. Can J Gastroenterol. 2007:21:25-9. DOI: 10.1155/2007/350279, PMID: 17225879

22 Takimoto K, Ueda T, Shimamoto F, Kojima Y, Fujinaga Y, Kashiwa A, et al. Sedation with dexmedetomidine hydrochloride during endoscopic submucosal dissection of gastric cancer. Dig Endosc. 2011;23:176-81. DOI: 10.1111/j.14431661.2010.01080.x, PMID: 21429025

23 Salinas FV, Sueda LA, Liu SS. Physiology of spinal anaesthesia and practical suggestions for successful spinal anaesthesia. Best Pract Res Clin Anaesthesiol. 2003;17:289-303. DOI: 10.1016/S1521-6896(02)00114-3, PMID: 14529003 